\title{
The Effect of Electric Cortical Stimulation after Focal Traumatic Brain Injury in Rats
}

\author{
Yong-Soon Yoon, Ph.D., Ki Pi Yu, M.D., Hyojoon Kim, Ph.D. ', Hyoung-ihl Kim, Ph.D. ' \\ Soo Hyun Kwak, M.D., Bong Ok Kim, M.D., Ph.D. ${ }^{2}$ \\ Department of Rehabilitation Medicine, Presbyterian Medical Center, ${ }^{1}$ Department of NeuroSurgery, \\ Presbyterian Medical Center, Jeonju 560-750, ${ }^{2}$ Department of Rehabilitation Medicine, School of Medicine, \\ Chungnam National University, Daejeon 301-721, Korea
}

\begin{abstract}
Objective To evaluate the effects of electric cortical stimulation in the experimentally induced focal traumatic brain injury (TBI) rat model on motor recovery and plasticity of the injured brain.

Method Twenty male Sprague-Dawley rats were pre-trained on a single pellet reaching task (SPRT) and on a Rotarod task (RRT) for 14 days. Then, the TBI model was induced by a weight drop device ( $40 \mathrm{~g}$ in weight, 25 $\mathrm{cm}$ in height) on the dominant motor cortex, and the electrode was implanted over the perilesional cortical surface. All rats were divided into two groups as follows: Electrical stimulation (ES) group with anodal continuous stimulation (50 Hz and $194 \mu$ s duration) or Sham-operated control (SOC) group with no electrical stimulation. The rats were trained SPRT and RRT for 14 days for rehabilitation and measured Garcia's neurologic examination. Histopathological and immunostaining evaluations were performed after the experiment.

Results There were no differences in the slice number in the histological analysis. Garcia's neurologic scores \& SPRT were significantly increased in the ES group $(\mathrm{p}<0.05)$, yet, there was no difference in RRT in both groups. The ES group showed more expression of c-Fos around the brain injured area than the SOC group.

Conclusion Electric cortical stimulation with rehabilitation is considered to be one of the trial methods for motor recovery in TBI. However, more studies should be conducted for the TBI model in order to establish better stimulation methods.
\end{abstract}

Key Words Cortical stimulation, Traumatic brain injury, Rehabilitation, Motor recovery

Received October 9, 2011; Accepted May 24, 2012

Corresponding author: Soo Hyun Kwak

Department of Rehabilitation Medicine, Presbyterian Medical Center, Sewon St. 68, Wansan-gu, Jeonju 560-750, Korea

Tel: +82-63-230-1468, Fax: +82-63-282-3385, E-mail: littlemicky@ hanmail.net

(c) This is an open-access article distributed under the terms of the Creative Commons Attribution Non-Commercial License (http:// creativecommons.org/licenses/by-nc/3.0) which permits unrestricted noncommercial use, distribution, and reproduction in any medium, provided the original work is properly cited.

Copyright $\odot 2012$ by Korean Academy of Rehabilitation Medicine

\section{INTRODUCTION}

Traumatic brain injury (TBI) occurs in various patterns depending on the patient's nationality, age, sex, and culture. It is commonly accompanied by the impairments of the physical, cognitive, and psychosocial functions. In addition, it also commonly occurs as a result of a car accident or a fall, or a trauma during exercise, and its occurrence is more than two times higher in men than in women. ${ }^{1}$ For patients with TBI, neurosurgical treatments should be made in the early period and rehabilitation 
should be accompanied afterwards. The methods of rehabilitation training are to recover the impaired brain function, to maximize residual function, to induce anatomical reconstruction of the brain, to use an orthosis or a device, and to reduce functional impairment by altering the environment. All of these treatment methods can be used concomitantly as well. ${ }^{1}$ In recent years, studies for gene and stem therapy are under investigation but are too far from becoming available in a clinical setting. Various types of pharmacological treatments have also been used but are of limited use to adjust the brain activity to a desirable extent. So in recent years, neuromodulation of brain has therefore become popular as additional rehabilitation that enhances brain plasticity. ${ }^{2}$

The neuromodulation of brain is advantageous in that it can be done with no respect to space and time and can suppress the competing brain regions by using inhibitory stimuli as well as stimulate the specific regions of the brain using excitatory stimuli; further, it can also be used with drug therapy. Moreover, it can achieve recovery of the brain function and brain plasticity through the treatment effect (after-effect). ${ }^{3-6}$ The electricity and magnetic fields are commonly used for neuromodulation therapy, and transcranial direct current stimulation (tDCS) and cortical electrical stimulation are representative methods for electrically stimulating the cerebral cortex. Of these, the electric cortical stimulation is advantageous in maintainining the duration of stimulus, stimulating the target region selectively, and being used in rehabilitation. ${ }^{7}$

Studies of cortical electrical stimulation were first attempted by Tsubokawa et al. ${ }^{8}$ in 1994 , who stimulated the motor cortex for treatment of chronic pain following the onset of stroke. These authors reported that some patients achieved recovery of the motor paralysis during treatment. Since then, many studies have been conducted. Up to the present, most of the studies on cortical electrical stimulation have examined the degree of recovery of motor function in an animal experimental model or in patients with cerebral infarction. Furthermore, electrical stimulation (ES) combined with rehabilitation training was found to result in motor functional improvements in many studies. ${ }^{9-13}$

Unlike cerebral infarction, TBI originates from injury to superficial layers of the cerebral cortex and then leads to diffuse the injury to white matter. With an increase in the acceleration and deceleration, it progresses to deep layers of the cerebral cortex. Though it has different patho- physiology from cerebral infarction, which occurs along the blood vessels, to this day, there are a few reports on animal experimental studies or clinical studies applying the cortical electrical stimulation therapy to TBI. Therefore, through an investigation, we aimed to establish baseline data for a variety of animal experimental studies on cortical electrical stimulation. In order to conduct the investigation, we inflicted trauma upon the motor area of the brain in rats and thereby created an animal experimental model of focal brain injury. We then examine the degree of recovery of the motor function and the brain plasticity after cortical electrical stimulation through rehabilitation.

\section{MATERIALS AND METHODS}

\section{Animals}

Twenty male Sprague-Dawley rats aged 120 days and weighing 250-300 grams (Samtako Biokorea, Daejeon, Korea) were used. They were housed in a transparent rat cage in the standard environment at a temperature of 21$24^{\circ} \mathrm{C}$ under a 12 hour light-dark cycle. All animal experiment protocols were carried out in accordance with the guidelines of animal experiments, edited by the Korean Academy of Medical Science (KAMS).

Group assignments of the animals: All rats were randomly divided into two groups; both groups were pretrained on a single pellet reaching task (SPRT) and rotarod task (RRT) for 14 days prior to the induction of brain injury. In both groups, TBI was induced. In the shamoperated control (SOC) group, an electrode was implanted and rehabilitation training was performed without electrical stimulation. In the electrical stimulation (ES) group, the rats received implantation of the cortical electrical stimulator electrode and received electrical continuous stimulation with rehabilitation training. To minimize the sacrifice of rats, behavioral test, neurological evaluation, and immunohistochemical staining was performed in serial order for each animal.

\section{Animal experimental model of post TBI using rats}

After all of the rats were given an intramuscular injection anesthesia with a mixture of ketamine hydrochloride (60 $\mathrm{mg} / \mathrm{kg}$ ) and xylazine hydrochloride $(7 \mathrm{mg} / \mathrm{kg})$, they were placed in a stereotaxic frame, the Model 900 Small animal stereotaxic instrument ${ }^{\circledR}$ (David Kopf Instruments, Tjunga, USA) adapting for their skull in a prone position. 
The rat's body temperature was maintained at $37.0 \pm 0.5^{\circ} \mathrm{C}$ by using the Homeothermic Blanket System ${ }^{\circledR}$ (Harvard apparatus Ltd, Edenbridge, UK). The cortical impact point was $1.0 \mathrm{~mm}$ anterior and $3.0 \mathrm{~mm}$ lateral to the bregma, which was the contralateral side of the dominant forelimb referred to rat brain map. ${ }^{14}$ After exposing $3 \mathrm{~mm}$ size of the rat's skull by a midline incision of $2.5 \mathrm{~cm}$, the dura mater was dissected and the impact was generated by dropping the $2 \mathrm{~mm}$ rounded piece of brass onto the rat's skull. TBI trauma device, the weight drop model, was applied according to the technique modified by Feeney et al., ${ }^{15}$ adapting the method of Ducker ${ }^{16}$ in order to induce a spinal cord injury. To induce TBI, the pieces of brass were dropped from a designated height through a stainless guided steel tube, which is perpendicular to the floor. Within the guided tube, we created small holes at a $1 \mathrm{~cm}$ gap in order to reduce the air resistance during the falling.
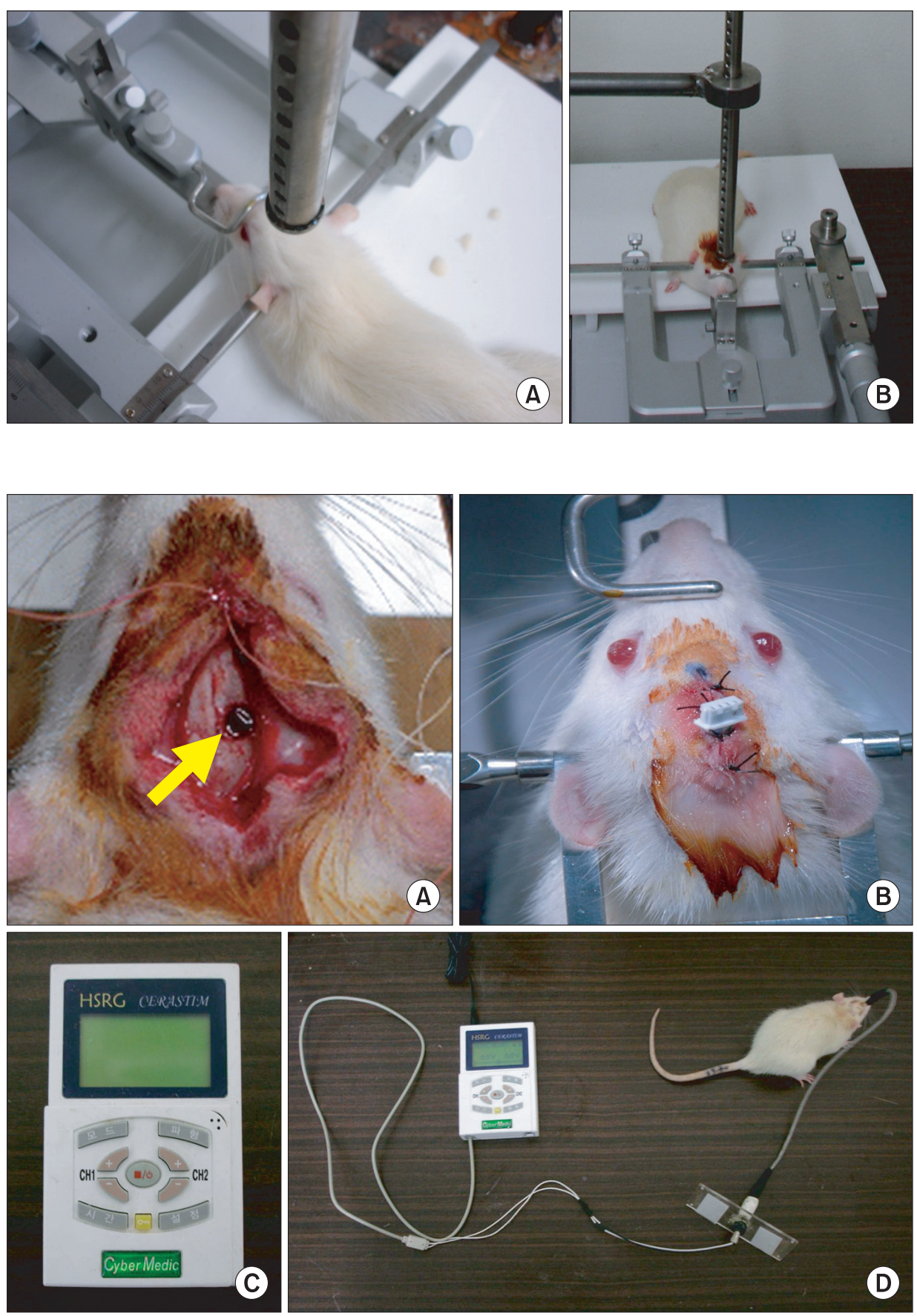

(D)
Fig. 1. (A) The guided tube was kept at a 90 degree angle and was perforated at $1 \mathrm{~cm}$ intervals to prevent air compression in the guided tube, (B) A free weight is released directly onto the exposed dura.
Fig. 2. (A) Circular injured lesion (arrow) in the right motor cortex of rat, (B) Implantation of stimulation electrode over the right hemisphere, (C) Stimulator for continuous electrical stimulation, and (D) Connection of stimulator and electrode in traumatic braininjured rat model. 
Since there is a tendency that rats, unlike humans, do not sustain a decreased motor function but achieve a good recovery, despite a certain level of injury, we have therefore attempted to trigger and examine a brain injury of moderate to severe degree. In order to do conduct such injury, we induced the dropping of a $40 \mathrm{~g}$ falling weight from a height of $25 \mathrm{~cm}$ at a pressure of $1.0 \mathrm{~kg} / \mathrm{cm}^{2}$ (Fig. 1). ${ }^{15}$

\section{Cortical electrical stimulation}

Electrode implantation for cortical stimulation: After the creation of the rat model of TBI, we applied the cortical electrical stimulation following the procedure by Yang et al. ${ }^{17}$ in a rat experimental model of cerebral infarction. A $3 \mathrm{~mm}$ diameter round bipolar stimulating electrode, made of stainless steel Oscor $^{\circledR}$ (Tampa, Florida, USA), was inserted to the anterior margin of the target site. The surface of the electrode covered the damaged brain tissue and its adjacent tissue on the dura mater. A $1 \mathrm{~mm}$ diameter stainless steel reference electrode was fixed $-1.5 \mathrm{~cm}$ (posterior) to the electrode on the skull. Both electrodes were fixed using polymethylmethacrylate (PMMA), and the skin edges were approximated around the implant except for the electrode and commutator system (Fig. 2).

Cortical electrical stimulation: After the creation of the rat model of brain injury with electrode implantation, the electrode cable was connected to HSRG Neuro ${ }^{\circledR}$ (Cybermedic Corp., Iksan, Korea), an electrical stimulator designed to select the intensity of stimulus, the time interval between the stimuli, and the frequency of stimulation and the anode (Fig. 2). In the ES group, the level of stimulation was determined to be set at the $50 \%$ of the motor threshold, which was defined as the lowest current to produce visible vibrissae movement. Starting from 0.8 $\mathrm{V}$, the motor threshold was measured once per two days, increasing the intensity by $0.1 \mathrm{~V}$. An electrical stimulator was designed to control the program, using the anodal electrical current of the pulse wave. The stimulator frequency was $50 \mathrm{~Hz}$ with pulse duration of 194 microseconds. Electrical stimulation was induced on the rats 24 hours a day from days 1 to 14 .

\section{Rehabilitation program}

Subsequent to the creation of the rat model of TBI, the SPRT and the RRT were performed once daily in the morning and in the afternoon for 14 days.

SPRT: The SPRT was conducted following the design adapted from Vergara-Aragon et al. ${ }^{18}$ Rats were pretrained to reach through the window in order to retrieve a single piece of food, a 45-mg single sucrose pellet (Bioserve Inc., Frenchtown, USA), for adaptation. Each rat performed the reaching trials 20 times each morning and in the afternoon for 20 min during the 14 days. The location of the food was reset to make the rats use their dominant forelimb. A clear Plexiglas box ( $35 \mathrm{~cm}$ long $\times 35$ $\mathrm{cm}$ wide $\times 45 \mathrm{~cm}$ high) was made for functional assessment and electrical stimulation, with the food shelf on the lateral side of the box $(5 \mathrm{~cm}$ long $\times 4 \mathrm{~cm}$ wide $\times 5 \mathrm{~mm}$ high) below the $1 \times 15 \mathrm{~cm}$ window, which is $2 \mathrm{~cm}$ from the floor to the top of the box (Fig. 3).$^{19}$ On post lesion day 1 , if the rats were successful in reaching at least one of the 20 trials, they were excluded from the current experiment because they were considered not to have enough brain injury of moderate to severe degree.

RRT: Following the creation of the rat model of TBI,
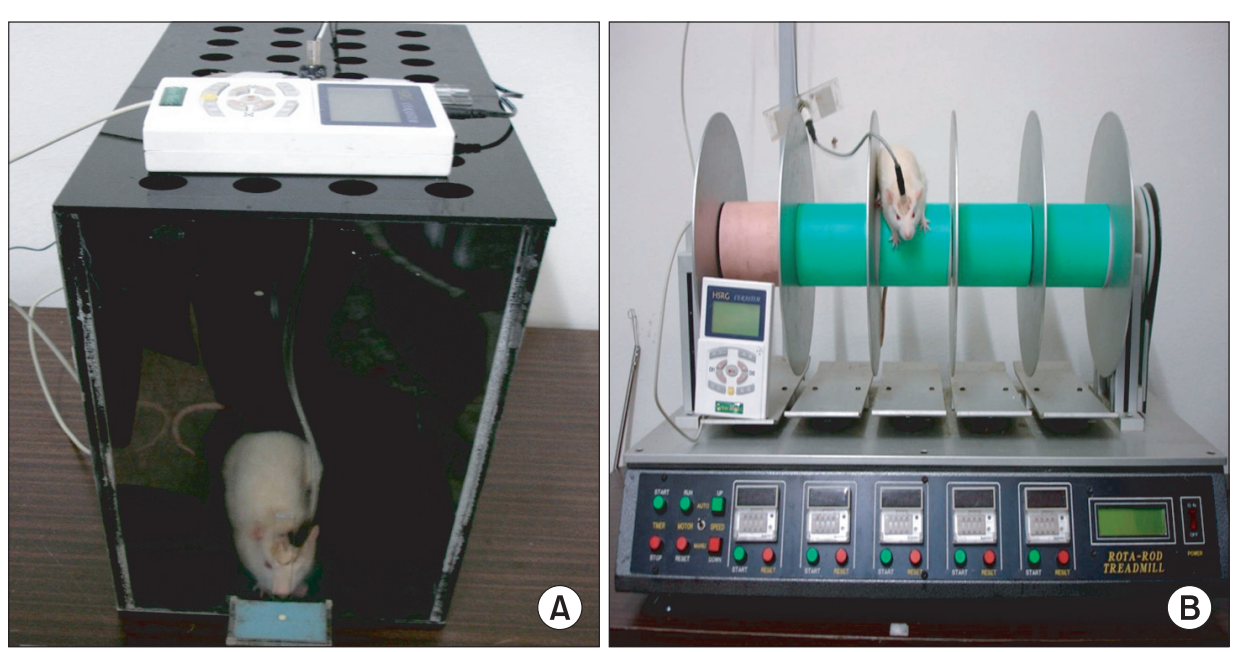

Fig. 3. (A) SPRT with electric cortical stimulation, (B) RRT with electric cortical stimulation. 
the rotarod task was performed to improve sensorimotor integration. This is based on the method proposed by Hunter et al. ${ }^{20}$ The rats were trained on the rotarod device consisting of five cylinders, in which the velocity was gradually accelerated from 4 to $40 \mathrm{rpm}$ for a maximum of 5 minutes. On the floor below the cylinder, there was a sensor that responded to the weight; thus, the training device was spontaneously stopped if the rat was dropped from the cylinder (Fig. 3). Animals were trained for 14 days three times each in the morning and in the afternoon, while receiving continuous electrical stimulation.

\section{Measurements}

We trained both the SPRT and RRT during a 14 day behavioral training period prior to the induction of the brain injury. The data was collected from two days prior to the preparation of the TBI rat model to the very day of the induction for baseline data (Fig. 4).

Neurological score: Neurological motor and sensory function were assessed in this study using the scoring system in rats with cerebral ischemic model, proposed by Garcia et al. ${ }^{21}$ The post-brain injury animals were scored by an examiner who was blind to the stimulation status at certain times at every other day from the post-brain injury date. The neurological score of Garcia et al. included the following six parameters: (1) Spontaneous activity, (2) Symmetry in the movement of four limbs, (3) Forepaw outstretching, (4) Climbing, (5) Body proprioception, and (6) Responses to vibrissae touch. Of the six param- eters, the first three have a minimum score of 1 , and the remaining three have 0 as their minimum score. In addition, all of the six parameters have a maximum score of 3 ; thus, if an animal is scored totally, the minimal neurological score is 3 and the maximum is 18 . If the rats obtained a score of more than 12 on the neurological scores proposed by Garcia et al., they were considered not to have brain injury of moderate or higher severity and were excluded from the experiment.

SPRT: The data from the afternoon session were chosen from the training sessions that were performed for 20 minutes each in the morning and in the afternoon. This analysis was based on the percentage of the success rate of rats reaching for food and that of the number of the pellets grasped with the forelimb and brought to the mouth without dropping, knocking, and missing. The scores were calculated based on the following formula: The success rate of SPRT (\%)=(The number of successful mouth consumption $/ 20) \times 100$.

RRT: Following the onset of the brain injury to the motor cortex in rats, the overall degree of functional recovery was assessed on the RRT. The mean time of the values of the 3 performances between pre and post-brain injury was calculated as \%.

The performance rate $(\%)=$ (The mean time of session of post-brain injury/The mean time of session of pre-brain injury) $\times 100$.

Histopathological study: After the end of the last training session, the rats were perfused transcardially with

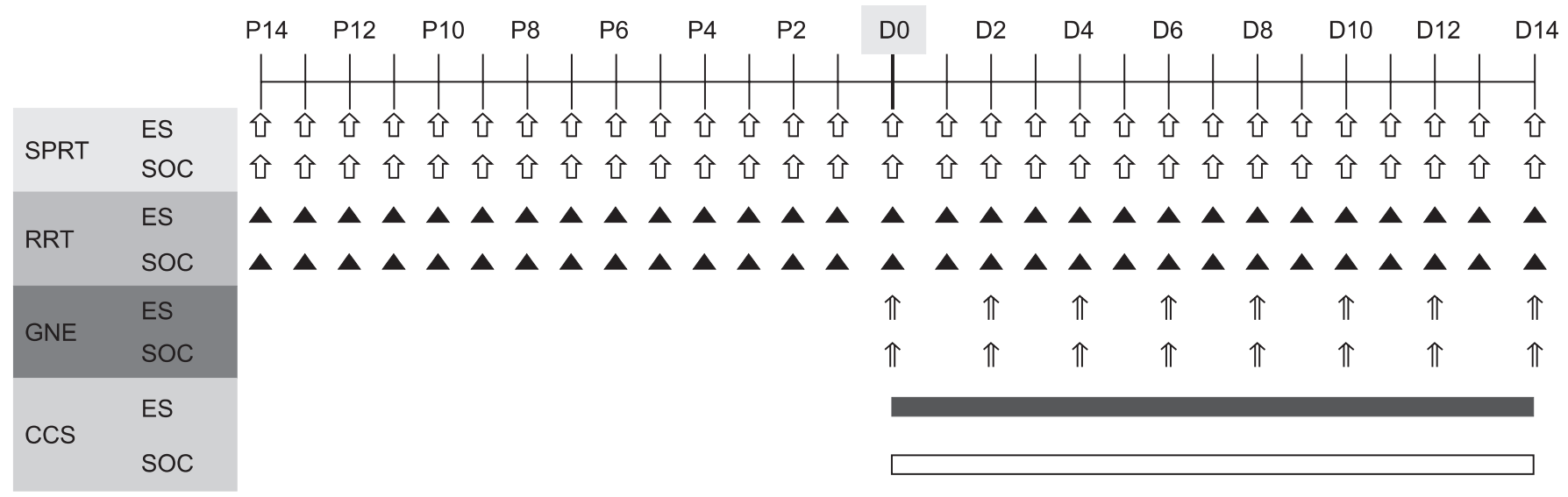

Fig. 4. Diagram of experimental design. After 14 days of preoperative training, all rats underwent traumatic brain injury in the motor cortex and implantation of stimulating electrode (D0). Only the experimental rats were trained with continuous electrical stimulation from day 1 (D1) to day 14 (D14) post-injury. P: Preoperative day, GNE: Garcia's neurologic examination, CCS: Continuous electric cortical stimulation, ES: Electric cortical stimulation group, SOC: Sham-operated control group. 
$1 \%$ paraformaldehyde (PFA), followed by $4 \%$ PFA fixatives. The brains were removed and were post-fixed in $4 \%$ paraformaldehyde (PFA) at $4^{\circ} \mathrm{C}$ for four hours and were subsequently replaced in $30 \%$ sucrose solution for more than 12 hours. The brain fixations were sectioned at a thickness of $40-\mu \mathrm{m}$ with a $400-\mu \mathrm{m}$ gap along the coronal plane using a Cryotome ${ }^{\circledR}$ (Thermo Fisher Scientific, Loughborough, UK). Tissue sections were then stained using a Hematoxylin (YD diagnostics, Yongin, Korea) and Eosin (PMC Histolab, Jeonju, Korea) dye mounted on a slide, which was smeared with poly-L-Lysine.

Immunohistochemical staining: To explore the tissue remodeling of the brain that mediates recovery of motor function in the impaired forelimb, we performed an immunohistochemistry. Among the selected, the coronal plane of $4 \mathrm{~mm}$ anterior and posterior to the center of the lesion, the $40 \mu \mathrm{m}$ sections, which was present on $+1.80 \mathrm{~mm}$, were used for lesion exploration. ${ }^{17}$ All tissue samples were immersed in distilled water for 30 minutes and were incubated with $0.3 \%$ hydrogen peroxide solution $\left(\mathrm{H}_{2} \mathrm{O}_{2}\right)$ in order to block the activity of endogenous peroxide. The sections were rinsed with Tris Buffer Solution (TBS) for 10 minutes and then permeabilized using $0.5 \%$ Triton X-100 ${ }^{\circledR}$ (Sigma-Aldrich, St. Louis, USA) for 30 minutes, and then rinsed again with TBS for 10 minutes. After 1 hour of immersion with TBS containing 5\% milk, c-Fos antibody (Calbiochem, Darmstadt, Germany) was applied to the sections for 24 hours at $4^{\circ} \mathrm{C}$, diluted in TBS of $10 \mu \mathrm{g} / \mathrm{ml}$ concentration. The sections were rinsed with TBS and then incubated with secondary Streptavidin Horseradish Peroxidase Conjugate antibody (Glostrup Denmark/Dakocytomation) for 20 minutes, 20-fold diluted for reaction, with ABC solution (Vector Laboratory Inc., Burlingame, USA) for 1 hour, and reacted with peroxide substrate solution (DAB) (Invitrogen Inc., Carnarillo, USA) for 5-10 minutes. The sections were rinsed with TBS between the reactions. The sections were washed with distilled water, dehydrated, immersed in xylene, and completely fixed with a mounting solution. The same sampling method was applied to the sections of the control group for comparison.

\section{Statistical analysis}

Statistical analysis was done using SPSS v.12.0 (SPSS Inc., Chicago, IL). Mann-Whitney tests were utilized in order to compare the volume of lesion, the number of tissue sections, neurological scores, and behavioral measures between the ES and the SOC groups. Wilcoxon signed ranks tests were used to examine the effect of treatment in each group. The significance levels were set at a p-value of $<0.05$.

\section{RESULTS}

\section{Focal TBI lesion in the cortex}

By 2 weeks post injury, all brain tissues sectioned from

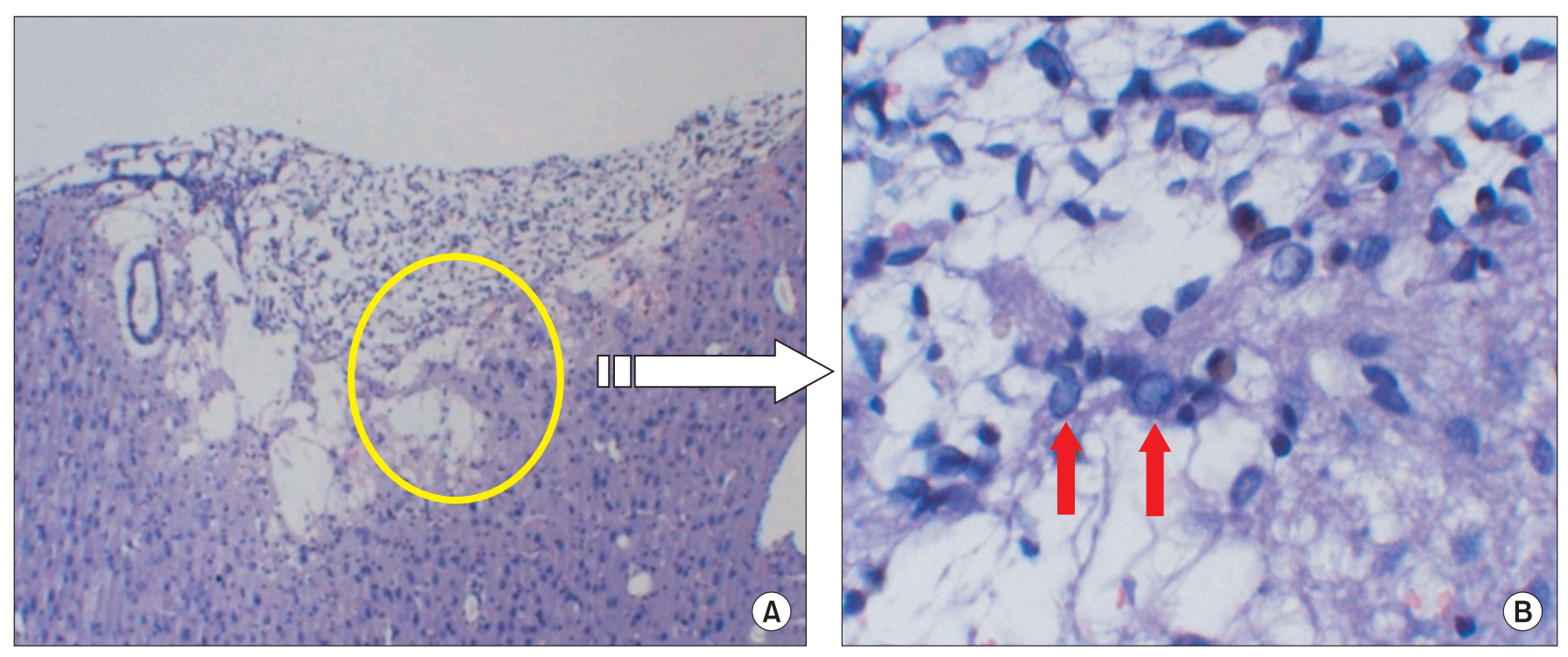

Fig. 5. The hematoxylin-eosin (HE) staining showed the traumatic injured area of the affected hemisphere; note the large cavity that involved the cortex at the site of injury (A), and the red arrow that shows the nuclear vacuolation in the brain cell after the traumatic brain injury (B). 
the rats had well developed trauma-induced lesions. On a microscopy, there were variable degrees of coagulation necrosis, cystic change within the necrotic tissue, and cavities due to the degeneration of the nucleus (Fig. 5). The number of tissue resections extracted from the braininjured site was measured and compared between the two groups, and no significant differences were observed in the volume of the lesion between the two groups (Table 1).

\section{Neurological scores}

In both the ES group and the SOC group, the neurological scores of Garcia et al. were improved as time elapsed. By day 6 post-brain injury, the ES group combined with the rehabilitation training had begun to show greater recovery compared to the SOC group $(\mathrm{p}<0.05)$ (Table 2) (Fig. $6)$.

\section{Changes in the SPRT scores}

Prior to the creation of the rat model of focal TBI, the mean success rate of SPRT showed no significant differences between the SOC group and the ES group. Following the brain injury, both groups showed an abrupt decrease in the SPRT scores to 0 points by post-lesion day 2 . In the ES group, the SPRT scores began to rise from postlesion day 3 and improved to $54.00 \pm 13.55 \%$ after 2 weeks later. In the SOC group, the SPRT scores were 0 points

Table 1. The Comparison of the Slice Number and the Total Volume of the Injured Area between the ES and SOC at 2 Weeks Post-injury

\begin{tabular}{lrrr}
\hline & ES $(\mathbf{n}=10)$ & SOC $(\mathbf{n}=10)$ & $\mathbf{p}$-value \\
\hline Injured volume $\left(\mathrm{mm}^{3}\right)$ & $32.00 \pm 2.79$ & $31.20 \pm 2.97$ & 0.786 \\
Total slice number & $9.70 \pm 1.06$ & $9.80 \pm 1.48$ & 0.823 \\
\hline
\end{tabular}

Values are mean \pm SD

ES: Electric cortical stimulation group, SOC: Sham-operated control group by post-lesion day 3 and were gradually improved starting at post-lesion day 4 to $18.00 \pm 10.06 \%$ after 2 weeks. The degree of recovery was significantly greater in the ES group compared to the SOC group from post-lesion day 5 $(p<0.05)$ (Table 3) (Fig. 7).

\section{Changes in the RRT scores}

Prior to the onset of brain injury, there were no significant differences in the mean value of the RRT score between the two groups ( $p>0.05$ ). During the 2 week post-injury observation period, by post-lesion day 3 , the mean value of RRT score was significantly greater in the SOC group but was lower at post-lesion day 6 compared with the ES group. However, this was not statistically significant. The mean value of RRT scores also observed no significant differences between the two groups ( $p>0.05$ ).

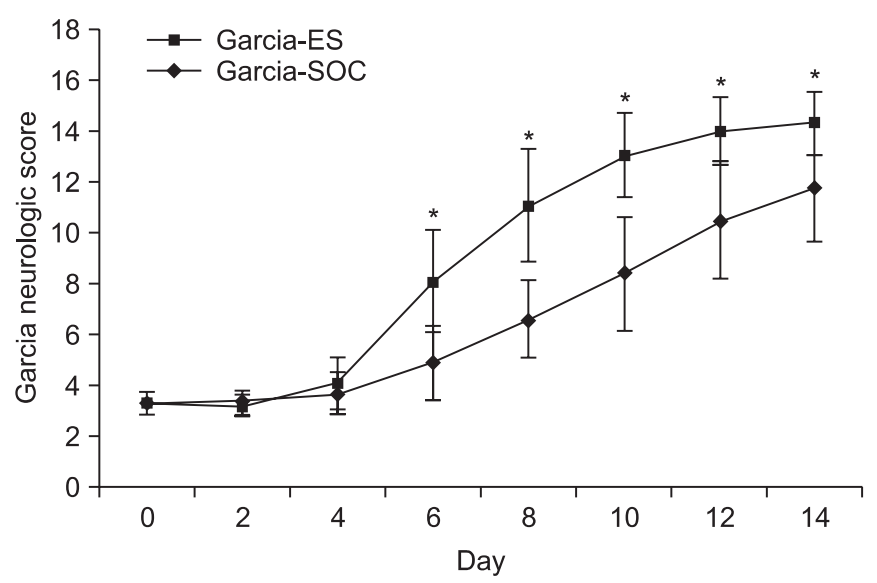

Fig. 6. Garcia's neurological score of the ES group and the sham-operated control group. There was a significant improvement in the ES group after the post-operation 6 days. Garcia-ES: Garcia neurological examination score of the electric cortical stimulation group, Garcia-SOC: Garcia neurological examination score of the shamoperated control group. * $\mathrm{p}<0.05$ by Mann-Whitney test between the two groups.

Table 2. Changes of the Garcia's Neurological Scores on Every Other Day after Traumatic Brain Injury

\begin{tabular}{lcccccccc}
\hline & D0 & D2 & D4 & D6 & D8 & D10 & D12 & D14 \\
\hline ES $(\mathrm{n}=10)$ & $3.33 \pm 0.42$ & $3.20 \pm 0.42$ & $4.10 \pm 0.99$ & $8.10 \pm 2.02^{*}$ & $11.10 \pm 2.23^{*}$ & $13.10 \pm 1.66^{*}$ & $14.00 \pm 1.33^{*}$ & $14.30 \pm 1.25^{*}$ \\
SOC $(\mathrm{n}=10)$ & $3.30 \pm 0.48$ & $3.30 \pm 0.48$ & $3.70 \pm 0.82$ & $4.90 \pm 1.45$ & $6.60 \pm 1.51$ & $8.40 \pm 2.27$ & $10.50 \pm 2.32$ & $11.80 \pm 2.15$ \\
\hline
\end{tabular}

Values are mean \pm SD

ES: Electric cortical stimulation group, SOC: Sham-operated control group, D: Date from traumatic brain injury from the very day (D0) to day 14 (D14)

${ }^{*} \mathrm{p}<0.05$ by Mann-Whitney test 
Table 3. Comparison of the SPRT and the RRT Scores between ES and SOC

\begin{tabular}{|c|c|c|c|c|c|c|}
\hline \multirow{2}{*}{ Day } & \multicolumn{3}{|c|}{ SPRT } & \multicolumn{3}{|c|}{ RRT } \\
\hline & $\operatorname{ES}(n=10)$ & $\operatorname{SOC}(n=10)$ & p-value* & ES $(n=10)$ & SOC $(n=10)$ & p-value* \\
\hline-2 & $78.00 \pm 3.54$ & $77.00 \pm 5.37$ & 0.901 & $72.00 \pm 12.31$ & $68.00 \pm 14.41$ & 0.427 \\
\hline-1 & $87.00 \pm 3.50$ & $87.00 \pm 4.22$ & 0.869 & $77.00 \pm 15.05$ & $69.00 \pm 10.23$ & 0.120 \\
\hline 0 & $88.00 \pm 4.22$ & $88.00 \pm 4.25$ & 0.745 & $77.00 \pm 10.11$ & $74.00 \pm 7.33$ & 0.494 \\
\hline 1 & 0 & 0 & 1.000 & $3.00 \pm 3.53$ & $3.00 \pm 3.96$ & 0.966 \\
\hline 2 & 0 & 0 & 1.000 & $6.00 \pm 4.11$ & $7.00 \pm 5.40$ & 0.819 \\
\hline 3 & $2.00 \pm 2.42$ & 0 & 0.067 & $10.00 \pm 4.65$ & $18.00 \pm 6.16$ & 0.059 \\
\hline 4 & $9.00 \pm 5.80$ & $5.00 \pm 2.84$ & 0.073 & $16.00 \pm 7.45$ & $20.00 \pm 5.13$ & 0.120 \\
\hline 5 & $19.00 \pm 9.94$ & $7.00 \pm 3.50$ & $0.002^{*}$ & $21.00 \pm 10.15$ & $25.00 \pm 10.00$ & 0.319 \\
\hline 6 & $28.00 \pm 14.58$ & $11.00 \pm 5.16$ & $0.001^{*}$ & $25.00 \pm 10.07$ & $23.00 \pm 8.82$ & 0.820 \\
\hline 7 & $35.00 \pm 18.26$ & $13.00 \pm 7.91$ & $0.001^{*}$ & $26.00 \pm 9.21$ & $24.00 \pm 9.82$ & 0.705 \\
\hline 8 & $37.00 \pm 14.18$ & $13.00 \pm 8.23$ & $<0.001^{*}$ & $26.00 \pm 9.59$ & $21.00 \pm 4.76$ & 0.172 \\
\hline 9 & $42.00 \pm 11.32$ & $14.00 \pm 7.47$ & $<0.001^{*}$ & $26.00 \pm 8.91$ & $24.00 \pm 6.67$ & 0.544 \\
\hline 10 & $43.00 \pm 9.50$ & $15.00 \pm 7.62$ & $<0.001^{*}$ & $24.00 \pm 8.97$ & $26.00 \pm 9.06$ & 0.850 \\
\hline 11 & $43.00 \pm 8.25$ & $16.00 \pm 8.43$ & $<0.001^{*}$ & $26.00 \pm 14.77$ & $25.00 \pm 4.58$ & 0.593 \\
\hline 12 & $48.00 \pm 8.88$ & $16.00 \pm 8.96$ & $<0.001^{*}$ & $27.00 \pm 9.54$ & $24.00 \pm 3.98$ & 0.819 \\
\hline 13 & $50.00 \pm 10.27$ & $18.00 \pm 9.50$ & $<0.001^{*}$ & $40.00 \pm 5.81$ & $37.00 \pm 6.29$ & 0.343 \\
\hline 14 & $54.00 \pm 13.55$ & $18.00 \pm 10.06$ & $<0.001^{*}$ & $58.00 \pm 5.82^{\dagger}$ & $53.00 \pm 9.72^{\dagger}$ & 0.103 \\
\hline
\end{tabular}

Values are mean \pm SD

ES: Electric cortical stimulation group, SOC: Sham-operated control group, SPRT: Single pellet reaching task, RRT: Rotarod task

${ }^{*} \mathrm{p}<0.05$ by Mann-Whitney test, ${ }^{\dagger} \mathrm{p}<0.05$ by Wilcoxon signed ranks test

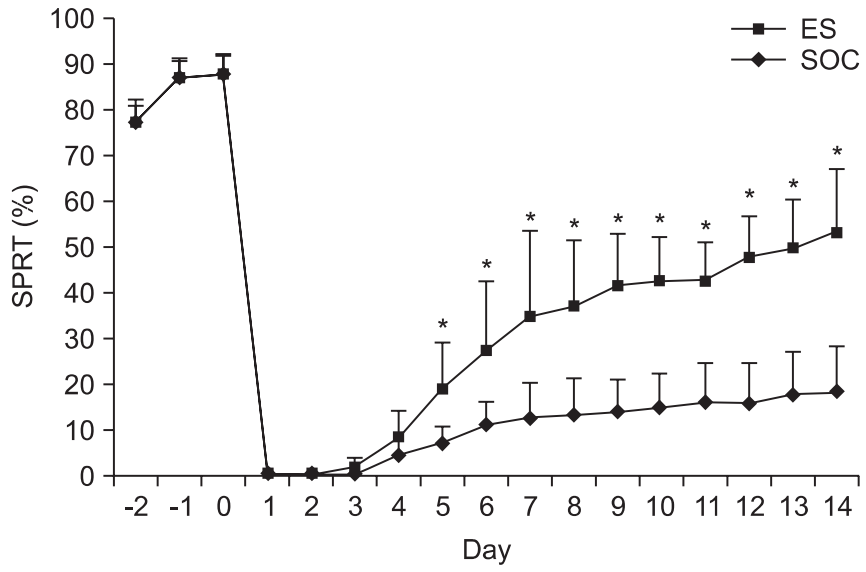

Fig. 7. Success rates of the single pellet reaching task (SPRT) of ES and SOC. The success rate of ES was higher than that of SOC from day 5 after the traumatic brain injury. ES: Electric cortical stimulation group, SOC: Shamoperated control group. ${ }^{*} \mathrm{p}<0.05$ by Mann-Whitney test between the two groups.

At 2 weeks post-injury, both ES and SOC groups exhibited significant recovery compared to post-lesion day 1 $(\mathrm{p}<0.05)$ (Table 3) (Fig. 8).

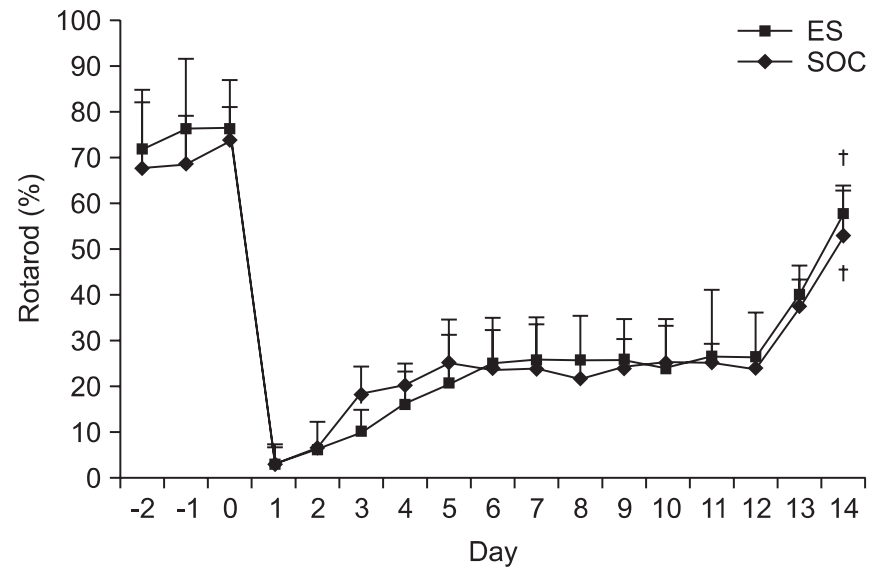

Fig. 8. The Rotarod task of ES and SOC. There were no significant differences for two weeks between ES and SOC but both groups showed general improvement afterwards. ES: Electric cortical stimulation group, SOC: Sham-operated control group. ${ }^{\dagger} \mathrm{p}<0.05$ by Wilcoxon signed ranks test.

Immunohistochemical findings

The expression of c-Fos on the injured motor cortex was observed in both the ES group and the SOC group. The degree of c-Fos expression was more marked in the 
ES group compared to the SOC group. The expression of c-Fos was also observed on the contralateral side of the lesion in the ES group, but showed less expression compared to the lesion side. In addition, it was predominantly found in the infragranular layer of the cerebral cortex (Fig. 9).

\section{Adverse effects during the experiment}

Following the electrical stimulation, there were no findings of motor disturbances in the head or extremities and severe spasticity. Also, there were no findings of epilepsy throughout the experiment period. Moreover, there were no findings of behavioral abnormalities due to the intracranial infections or abscess on the histopathologic evaluations following the electrode implantation.

\section{DISCUSSION}

There are various types of neuromodulatory treatment that are performed for patients with brain injury. Of these, transcranial magnetic stimulation (TMS), transcranial direct current stimulation (tDCS), cortical electrical stimulation (ES), and deep brain stimulation (DBS) are performed to mediate the recovery of the brain function with direct stimulation of the brain, using the mainly electrical current or magnetic field. Although cortical ES has a disadvantage of relatively high invasiveness compared with the other methods, requiring surgical operation under general anesthesia, it is the most excellent method in the perspective of energy efficiency, accuracy of stimulation, and the possibility of combining with rehabilitation. ${ }^{22}$

To date, most studies about cortical ES have been performed related to cerebral infarction. Brown et al. ${ }^{13}$ reported that the ES of motor cortex enhances brain function, promotes cortical reorganization, and blocks inhibitory action in the subcortical level. In addition, Kleim et al. ${ }^{9}$ and Plautz et al. ${ }^{10}$ reported that the cortical ES can drive brain plasticity and potentiate a new network instead of a damaged one. ${ }^{23,24}$

According to the study about the frequency for the cortical ES of Adkins-Muir and Jones, ${ }^{25}$ a rat model of cerebral infarction divided into $50 \mathrm{~Hz}$ stimulation and 250 $\mathrm{Hz}$ stimulation groups showed greater rates of improve-
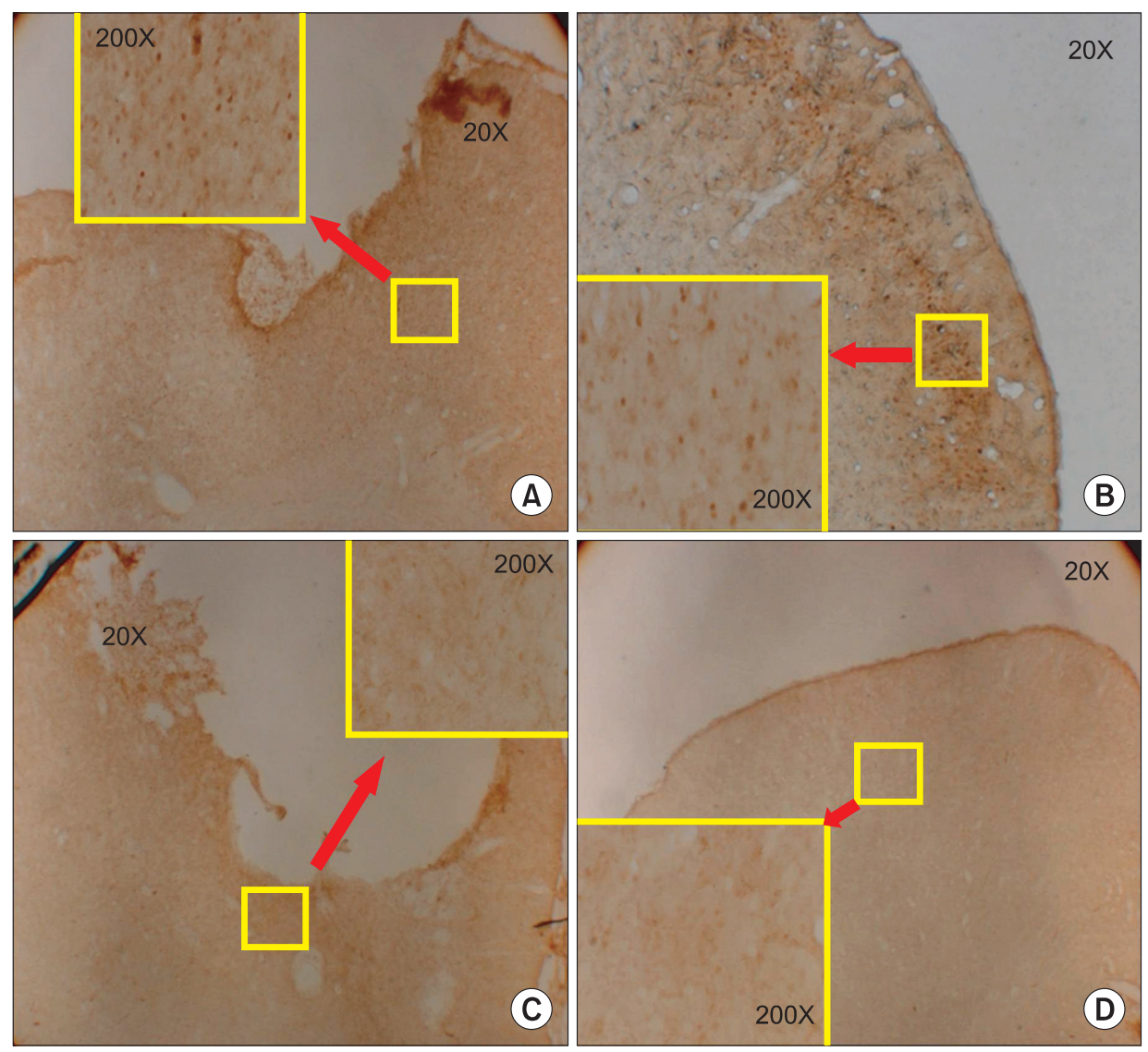

Fig. 9. Immunohistochemical staining for c-Fos protein in the motor cortex. (A) The ipsilesional motor cortex of the electric cortical stimulation group, (B) the contralateral motor cortex of the electric cortical stimulation group, (C) the ipsilesional motor cortex of the sham-operated control group, and (D) the contralateral motor cortex in the sham-operated control group. Note the increase of c-Fos expression in the perilesional area of the motor cortex of the electric cortical stimulation group and (A) c-Fos expression in the infragranular cell layer of the contralateral cortex (B). 
ment with the impaired forelimb in the $50 \mathrm{~Hz}$ stimulation group, but showed no significant improvement in the $250-\mathrm{Hz}$ stimulation group. Also, Teskey et al. ${ }^{26}$ found that the group that received $50 \mathrm{~Hz}$ to $100 \mathrm{~Hz}$ showed significantly greater efficacy in the rat model of cerebral infarction. Therefore, in the present study, we used the $50 \mathrm{~Hz}$ frequency stimulation.

According to the study of Moon et al. ${ }^{27}$ on continuous stimulation (CS) and intermittent stimulation (IS) in a rat model of cerebral infarction, CS showed better results in the animal group with severe infarction, whereas IS showed greater recovery in the animal group with infarction of lower to moderate severity. These authors assumed that the CS in the rat model of moderate degree infarction might induce additional injuries in the cerebral neurons of the penumbra area. However, this might be minimized following the IS because there was a resting period in the acute and subacute period. These authors also noted that prolonged stimulation was required, rather than minimizing additional injury in the acute period, in order to recruit the neural plasticity, because the severe degree infarction rat model group lacked of the remaining plastic motor cortex due to irreversible injury. We therefore used the continuous stimulation in an animal model of moderate to severe degree brain injury in the current study.

Rat models of TBI include weight drop model, controlled cortical impact (CCI) model, and fluid percussion injury (FPI) model. ${ }^{28,29}$ Of these, the weight drop model is inexpensive, easy to perform requiring simple devices, and can reproduce brain edema and contusion, which replicates TBI that occurs in a clinical setting. However, it has a disadvantage of not being able to quantify the severity of the brain injury. The CCI model has advantages such that it can quantify the impacts causing brain injuries and can also prevent the occurrence of injuries due to repulsive force. However, it can reproduce insufficient impact to induce the brain edema and contusion, needing longer time of the impacts and thus, it may not trigger a severe brain injury. The FPI model is a method for inflicting brain injuries by application of a fluid pressure pulse. Advantages of the FPI model are that the magnitude, velocity, and intensity of the impacts can be controlled accurately; yet, the disadvantage is that air in the cylinder can generate resistance so that the acceleration is not reflected. Therefore, in our study, we dropped the same gram of weight from the same height in order to compensate the demerits of the weight drop model, which can replicate the most natural type of TBI. In addition, we modified our guided tube with holes at a $1-\mathrm{cm}$ gap in order to reduce air resistance as much as possible. ${ }^{15}$

Since the methods for evaluation of a specific function in a TBI rat model have not been found in previous reports, we used the methods of that which evaluate the functions in a rat model of cerebral infarction. Methods for evaluating the function in a rat model of cerebral infarction include the neurological evaluations of Garcia, Tray reaching task (TRT), ${ }^{30}$ Single pellet reaching task (SPRT), ${ }^{30}$ sunflower seed-eating test, ${ }^{31}$ cylinder test, ${ }^{32}$ swimming test, ${ }^{33}$ and tongue protrusion test. ${ }^{34}$ Neurological evaluations of Garcia have been used to examine as to whether there is a cerebral infarction in a rat model of cerebral infarction, ${ }^{35,36}$ whose results can be graded based on numerical scores. They also contain the assessment of the functions of the forelimb. In this study, we assessed whether TBI was made with the neurological scores of Garcia, and we also examined the degree of recovery with that. Such methods of sunflower seed-eating test, cylinder test, swimming test, and tongue protruding test have a disadvantage as to the great discrepancy in the test outcomes resulting from the subjective judgment of the observers. They also require not only a complicated device but also a large place for the installation of the device. By contrast, TRT, SPRT, and RRT can be performed even with the use of a simple device in a small space. They also have the advantage of being able to use objective scores for the assessment. Among them, Kim et al.$^{37}$ found that TRT reflected the functional deficit in the early stage of the brain injury, yet it did not correlate with the outcomes of functional recovery. As a result, we did not use TRT for the functional assessment in this study. Although RRT was not sufficient to obtain the constant data, we used RRT in order to examine the overall pattern of functional recovery and the changes in the sensory and motor functions because we developed a moderate to severe graded injured rat model. Kim et al. ${ }^{37}$ found that SPRT was one of the most appropriate methods for assessing the recovery of function following the loss of brain function after brain injury. ${ }^{38}$ SPRT was closely correlated with the magnetic resonance imaging (MRI) and histopathological findings; it further reflected the functional outcomes in the 
rat model of cerebral infarction. Hence, we used SPRT for the functional assessment in this study.

In our study, we have conducted the RRT in order to assess the motor coordination functions, equilibrium, and motor learning ability. It requires a high level of integrated motor functions with proprioception, balance, delicate motor control, perception of height, and anxiety. It is known that RRT has a lack of consistency in the previous results in the animal experimental studies. ${ }^{37}$ This lack of consistency might be due to the ability of the experimental animals adapting to the environment as well as multiple factors, such as the anxiety level and balance, having an effect on the assessment. Yet, in our study, the RRT was performed in order to see the changes in higher motor functions after TBI. We have observed the overall recovery of brain injury in both groups. Recovery in higher motor functions might be followed after the recovery of the brain injury. The differences of functional recovery between the ES group and SOC group might have not fully exhibited with the short duration of training because the rat's higher motor function, including concentration and balance capability, were the major variables for the RRT test. Besides, the ability of height perception, anxiety level, and equilibrium in each rat can affect the RRT test; therefore, the accurate comparison about difference of the groups was not examined in this study. Future studies will be required to observe more accurate outcomes, using long-term training, which can restore the connections between proprioception and motor functions and equilibrium and motor functions with the overall recovery of the motor function.

c-Fos is one of the genes that is expressed in the cell when tissue injury and inflammation is induced. It is promptly expressed in the post-synaptic neurons in response to various stimuli. Hence, due to the sensitivity, it is used as a neurological marker for the activation of neurons in the brain and spinal cord following the central nervous system (CNS) injury. ${ }^{39}$ As to the immunohistochemistry in the current study, the ES group exhibited an increased expression of c-Fos around the brain lesion more than the other group. Presumably, the cranial neurons might be enhanced to recover more in the ES group compared with the SOC group. The expression of c-Fos was also observed on the contralateral hemisphere of the brain lesion. In view of our findings, ES can affect the contralateral hemisphere so that it may cause c-Fos expression, or ES can induce the activation of the contralateral hemisphere. A majority of c-Fos expression on the contralateral side of the lesion is primarily shown in the infragranular layer of the brain. Considering that pyramidal cells are mainly distributed in the infragranular layer in the brain and descending fibers are sprouted to the brain stem or spinal cord, the activation of the lesion by stimulation might affect the contralateral side.

A limitation of this study was the method of producing a TBI model of the brain. Since we followed an experimental model of focal TBI by dissecting the skull and inducing a direct injury to the brain, the results of this study did not accurately reflect the clinical TBI state with the widespread diffuse axonal injury component combined to focal injury by moderate to severe impact on the brain. Further study will be useful to observe the various outcomes if the diffuse axonal injury is produced by the impact on the brain without skull excision. This study was conducted to observe the 14 day-outcome after cortical ES combined with rehabilitation training in a TBI rat model. Moreover, further complemented studies will be required to determine the specific time points when the ES has effects in achieving the recovery of functions on pathophysiology. This should also be accompanied by long-term follow-up studies.

Furthermore, it is also needed that further trials should be conducted to identify the most appropriate methods of ES based on the various frequency, duration of stimulation, and the types of rehabilitation training following the creation of an animal experimental model of TBI by applying various methods and intensity.

\section{CONCLUSION}

In this study, we investigated the degree of recovery through the evaluation of functional test after undergoing the cortical ES combined with rehabilitation training. In addition, we also examined the brain plasticity using histopathologic examination and immunohistochemical staining. Our results showed that in rat models of focal TBI, there was a significant recovery in the ES group that received the cortical ES combined with rehabilitation training, compared to the SOC group receiving training alone. Also, there was a significant recovery in the neurological scores of Garcia and in the behavioral test based on the SPRT. However, there was no significant difference 
in the RRT that indicated the sensory and motor functions between the two groups.

Together, these findings suggest that electrical cortical stimulation combined with rehabilitation training enhances the improvement of motor function impairments and also enhances plasticity of the injured brain lesion.

\section{ACKNOWLEDGEMENTS}

This study was supported by a grant of the Korea of Healthcare Technology R\&D Project, Ministry for Health \& Welfare \& Family Affairs, Republic of Korea (A091220).

\section{REFERENCES}

1. Cifu DX, Kreutzer JS, Slaster DN, Tayor L. Rehabilitation after traumatic brain injury. In: Braddom RL, Editor. Physical medicine and rehabilitation, 3rd ed, Philadelphia: Saunders, 2006, 1133-1174

2. Walsh V, Desmond JE, Pascual-Leone A. Manipulating brains. Behav Neurol 2006; 17: 131-134

3. Fregni F, Pascual-Leone A. Technology insight: noninvasive brain stimulation in neurology-perspectives on the therapeutic potential of rTMS and tDCS. Nat Clin Pract Neurol 2007; 3: 383-393

4. Alonso-Alonso M, Fregni F, Pascual-Leone A. Brain stimulation in poststroke rehabilitation. Cerebrovasc Dis 2007; 24 Suppl 1: 157-166

5. Pascual-Leone A. Disrupting the brain to guide plasticity and improve behavior. Prog Brain Res 2006; 157: 315-329

6. Harris-Love ML, Cohen LG. Noninvasive cortical stimulation in neurorehabilitation: a review. Arch Phys Med Rehabil 2006; 87: S84-93

7. Harvey RL, Nudo RJ. Cortical brain stimulation: a potential therapeutic agent for upper limb motor recovery following stroke. Top Stroke Rehabil 2007; 14: 5467

8. Tsubokawa T, Katayama Y, Yamamoto T, Hirayama T, Koyama S. Chronic motor cortex stimulation for the treatment of central pain. Acta Neurochir Suppl 1991; 52: 137-139

9. Kleim JA, Bruneau R, VandenBerg P, MacDonald E, Mulrooney R, Pocock D. Motor cortex stimulation enhances motor recovery and reduces peri-infarct dysfunction following ischemic insult. Neurol Res 2003;
25: 789-793

10. Plautz EJ, Barbay S, Frost SB, Friel KM, Dancause N, Zoubina EV, Stowe AM, Quaney BM, Nudo RJ. Postinfarct cortical plasticity and behavioral recovery using concurrent cortical stimulation and rehabilitative training: a feasibility study in primates. Neurol Res 2003; 25: 801-810

11. Katayama Y, Tsubokawa T, Yamamoto T. Chronic motor cortex stimulation for central deafferentation pain: experience with bulbar pain secondary to Wallenberg syndrome. Stereotact Funct Neurosurg 1994; 62: 295299

12. Brown JA, Lutsep H, Cramer SC, Weinand M. Motor cortex stimulation for enhancement of recovery after stroke: case report. Neurol Res 2003; 25: 815-818

13. Brown JA, Lutsep H, Weinand M, Cramer SC. Motor cortex stimulation for the enhancement of recovery from stroke: a prospective, multicenter safety study. Neurosurgery 2006; 58: 464-473

14. Paxinos G, Watson C. The rat brain in stereotaxic coordinates, 6th ed, London: Elsevier, 2007, 30-82

15. Feeney DM, Boyeson MG, Linn RT, Murray HM, Dail WG. Response to cortical injury: I. Methodology and local effects of contusions in the rat. Brain Res 1981; 211: 67-77

16. Ducker TB. Experimental injury of the spinal cord. In: Vinken PJ, Bruyn GW, Editors. Handbook of clinical neurology, 1st ed, Amsterdam: North-Hollan Publishing Co., 1976, 9-26

17. Yang CY, Moon SK, Song JH, Kim HS, Han EH, Kim TJ, Shin YI. The effect of continuous epidural electrical stimulation on synapse and neuronal cell in rat with focal ischemia. J Korean Acad Rehab Med 2008; 32: 375-387

18. Vergara-Aragon P, Gonzalez CL, Whishaw IQ. A novel skilled-reaching impairment in paw supination on the "good" side of the hemi-Parkinson rat improved with rehabilitation. J Neurosci 2003; 23: 579-586

19. Moon SK, Yang CY, No SE, Kim EY, Lee S, Park SA, Oh GJ, Kim HI, Song JH, Lee MC, et al. Promotion of motor recovery by anodal continuous and low amplitude cortical stimulation in rat stroke model. Lab Anim Res 2007; 23: 25-30

20. Hunter AJ, Mackay KB, Rogers DC. To what extent have functional studies of ischemia in animals been useful in the assessment of potential neuroprotective 
agents? Trends Pharmacol Sci 1998; 19: 59-66

21. Garcia JH, Wagner S, Liu KF, Hu XJ. Neurological deficit and extent of neuronal necrosis attributable to middle cerebral artery occlusion in rats. Statistical validation. Stroke 1995; 26: 627-635

22. Cogiamanian F, Marceglia S, Rossi L, Torre ED, Priori A. Transcranial magnectic and direct current stimulation: a primer. In: Canavero S, Editor. Textbook of therapeutic cortical stimulation, NewYork: Nova Science Publishers, 2009, 45-56

23. Sawaki L, Wu CW, Kaelin-Lang A, Cohen LG. Effects of somatosensory stimulation on use-dependent plasticity in chronic stroke. Stroke 2006; 37: 246-247

24. Kreisel SH, Hennerici MG, Bazner H. Pathophysiology of stroke rehabilitation: the natural course of clinical recovery, use-dependent plasticity and rehabilitative outcome. Cerebrovasc Dis 2007; 23: 243-255

25. Adkins-Muir DL, Jones TA. Cortical electrical stimulation combined with rehabilitative training: enhanced functional recovery and dendritic plasticity following focal cortical ischemia in rats. Neurol Res 2003; 25: 780-788

26. Teskey GC, Flynn C, Goertzen CD, Monfils MH, Young NA. Cortical stimulation improves skilled forelimb use following a focal ischemic infarct in the rat. Neurol Res 2003; 25: 794-800

27. Moon SK, Shin YI, Kim HI, Kim HJ, Lee JO, Lee MC. Effect of prolonged cortical stimulation differs with size of infarct after sensorimotor cortical lesions in rats. Neuroscience lett 2009; 460: 152-155

28. Prins ML, Hovda DA. Developing experimental models to address traumatic brain injury in children. J Neurotrauma 2003; 20: 123-137

29. Park HK, Fernandez II, Dujovny M, Diaz FG. Experimental animal models of traumatic brain injury: medical and biomechanical mechanism. Crit Rev Neurosurg 1999; 9: 44-52
30. Gharbawie OA, Gonzalez CLR, Whishaw IQ. Skilled reaching impairment from the lateral frontal cortex component of middle cerebral artery stroke: a qualitative and quantitative comparison to focal motor cortex lesions in rat. Behav Brain Res 2005; 156: 125-137

31. Whishaw IQ, Sarna JR, Pellis SM. Evidence for rodentcommon and species-typical limb and digit use in eating, derived from a comparative analysis of ten rodent species. Behav Brain Res 1998; 96: 79-91

32. Schallert T, Kozlowski DA, Humm JL, Cocke RR. Use dependent structural events in recovery of function. Adv Neurol 1997; 73: 229-238

33. Gonzalez CL, Kolb B. A comparison of different models of stroke on behavior and brain morphology. Eur J Neurosci 2003; 18: 1950-1962

34. Whishaw IQ, Kolb B. "Stick out your tongue": tongue protrusion in neocortex and hypothalamus damaged rats. Physiol Behav 1983; 30: 471-480

35. Choi YB, Kim YI, Lee KS, Kim BS, Kim DJ. Protective effect of epigallocatechin gallate on brain damage after transient middle cerebral artery occlusion in rats. Brain Res 2004; 1019: 47-54

36. Kim DY, Park SH, Lee SU, Choi DH, Park HW, Paek SH, Shin HY, Kim EY, Park SP, Lim JH. Effect of human embryonic stem cell-derived neuronal precursor cell transplantation into the cerebral infarct model of rat with exercise. Neurosci Res 2007; 58: 164-175

37. Kim HS, Shin YI, Kim HI, Moon SK, Lee JO, Moon BS, Lee MC. Relevance of behavioral test in the photothrombotic stroke rat model. J Korean Acad Rehab Med 2006; 30: 135-141

38. Gonzalez CLR, Kolb B. A comparison of different models of stroke on behavior and brain morphology. Eur J Neurosci 2003; 18: 1950-1962

39. Dubner R, Ruda MA. Activity-dependent neuronal plasticity following tissue injury and inflammation. Trends Neurosci 1992; 15: 96-103 